PROCEEDINGS OF THE

AMERICAN MATHEMATICAL SOCIETY

Volume 127, Number 12, Pages 3493-3494

S 0002-9939(99)05009-1

Article electronically published on May 17, 1999

\title{
TORIC DEGENERATIONS AND VECTOR BUNDLES
}

\author{
JOSEPH GUBELADZE \\ (Communicated by Wolmer V. Vasconcelos)
}

\begin{abstract}
There are many affine subalgebras of polynomial rings with highly non-trivial projective modules, whose initial algebras (toric degenerations) are still finitely generated and have all projective modules free.
\end{abstract}

Let $k\left[X_{1}, \ldots, X_{n}\right]$ be a polynomial algebra ( $k$ a field, $n \in \mathbb{N}$ ) and $A$ an affine $k$-subalgebra. Let $\prec$ denote a term order on the multiplicative semigroup of monomials in the $X_{i}$ and let $\operatorname{in}_{\prec}(A)$ denote the monomial subalgebra of $k\left[X_{1}, \ldots, X_{n}\right]$, generated by the leading monomials of elements $f \in A$ with respect to $\prec$. In case the initial algebra $\operatorname{in}_{\prec}(A)$ is finitely generated, one can obtain many properties of $A$ by checking them for $\operatorname{in}_{\prec}(A)$ (called sometimes a toric degeneration of $A$ ) (see $[\mathrm{CHV}],[\mathrm{RS}]$ ). However, this is not the case for the property 'all projective modules are free' - thanks to Bernd Sturmfels for asking me this question.

Theorem 1. Let $A=k\left[X, Y, Z^{2}, Z^{3}-X Y Z\right]$ and $\prec$ be the lexicographic term order corresponding to $Z \prec Y \prec X$. Then $S K_{0}(A)=\operatorname{Ker}\left(\widetilde{K}_{0}(A) \stackrel{\text { det }}{\rightarrow} \operatorname{Pic}(A)\right)$ is not trivial (equivalently, there are projective $A$-modules which are not even stably of type free $\oplus$ rank 1), while $\operatorname{in}_{\prec}(A)$ is finitely generated and all projective $\operatorname{in}_{\prec}(A)$-modules are free.

(Here 'projective' includes 'finitely generated'.)

Proof. Since $\left(Z^{2}-X Y\right) k[X, Y, Z] \subset A$, the following diagram with the upper horizontal identity embedding is a pull-back diagram (all the letters refer to variables)

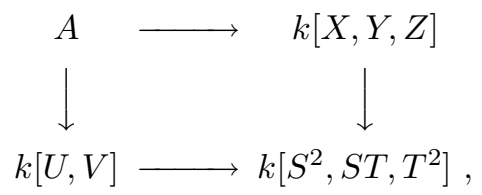

where $X \mapsto S^{2}, Y \mapsto T^{2}, Z \mapsto S T, U \mapsto S^{2}, V \mapsto T^{2}$. It is similarly easy to show that $\operatorname{in}_{\prec}(A)=k\left[X, Y, Z^{2}, X Y Z\right]$ - a seminormal monomial algebra (as a $k$ vector space it is generated by the normal monomial subalgebras $k[X, Y], k\left[X, Z^{2}\right]$, $k\left[Y, Z^{2}\right]$ and $\left.k\left[\left\{X^{a} Y^{b} Z^{c} \mid a>0, b>0, c>0\right\}\right]\right)$. So by [Gu1] projective modules over in $\prec(A)$ are free, while the Mayer-Vietoris sequence (see [Bass], p.490), applied to the diagram above, implies $S K_{0}(A)=S K_{1}\left(k\left[S^{2}, S T, T^{2}\right]\right)$. Hence, by [Gu2] $S K_{0}(A) \neq 0$.

Received by the editors February 20, 1998.

1991 Mathematics Subject Classification. Primary 13D15, 19A49.

This research was supported in part by the Alexander von Humboldt Foundation and CRDF grant \#GM1-115. 
Remark 2. Actually, by the (U - Pic)-Mayer-Vietoris sequence $\operatorname{Pic}(A)=0$ for the ring $A$ in Theorem 1.

By [Gu2] essentially all (and conjecturely all) monomial algebras, not isomorphic to polynomial rings, have non-trivial $S K_{1}$-groups. So changing the lower right algebra in the diagram above by other monomial algebras and changing correspondingly the 'side' polynomial algebras, one can produce a big class of rings having this property. One uses the general observation that if some powers of all variables belong to $A$, both $A$ itself and its initial algebra in $_{\prec}(A)$ are finitely generated because they are intermediate algebras of the module finite extensions

$$
k\left[X_{1}^{a_{1}}, \ldots, X_{n}^{a_{n}}\right] \subset k\left[X_{1}, \ldots, X_{n}\right] .
$$

Nontriviality of $S K_{1}$ for the special monomial ring $k\left[S^{2}, S T, T^{2}\right]$ (over a characteristic zero field $k$ ) was also shown in [Sri].

\section{REFERENCES}

[Bass] H. Bass, Algebraic K-theory, W. A. Benjamin, Inc., 1968. MR 40:2736

[CHV] A. Conca, J. Herzog, G. Valla, SAGBI bases with applications to blow-up algebras, J. Reine Angew. Math. 474 (1996), 113-138. MR 97h:13023

[Gu1] J. Gubeladze, Anderson's conjecture and the maximal monoid class, over which projective modules are free, Math. USSR Sbornik 63 (1989), 165-180. MR 89d:13010

[Gu2] J. Gubeladze, Nontriviality of $S K_{1}(R[M])$, J. Pure Appl. Alg. 104 (1995), 169-190. MR 96j:19002

[RS] L. Robbiano and M. Sweedler, Subalgebra bases, in Commutative algebra, Proc. Workshop, Salvador/Brasil 1988, Lect. Notes Math. 1430 (1990), 61-87. MR 91f:13027

[Sri] V. Srinivas, $K_{1}$ of the cone over a curve, J. Reine Angew. Math. 381 (1987), 37-50. MR 89e: 14008

A. Razmadze Mathematical Institute, Alexidze St. 1, 380093 Tbilisi, Georgia

E-mail address: gubel@rmi.acnet.ge 